\title{
Treatment of fibrous dysplasia of the zygomaticomaxillary complex with radical resection and three-dimensional reconstruction with autologous calvarial bone graft
}

\author{
Sung Jae Ahn ${ }^{1}$, \\ Jong Won Hong ${ }^{1,2}$, \\ Yong Oock Kim ${ }^{1,2}$, \\ Dae Hyun Lew ${ }^{1,2}$, \\ Won Jai Lee ${ }^{1,2}$ \\ ${ }^{1}$ Department of Plastic and \\ Reconstructive Surgery and ${ }^{2}$ Institute \\ for Human Tissue Restoration, Yonsei \\ University College of Medicine, Seoul, \\ Korea
}

\begin{abstract}
Fibrous dysplasia (FD) is a rare, benign bone disease with abnormal bone maturation and fibroblastic proliferation. Optimal treatment of zone 1 craniofacial FD is radical resection and reconstruction. To achieve of structural, aesthetic, and functional goals, we use three-dimensionally designed calvarial bone graft for reconstruction of zygomatic defect after radical resection of FD. The authors used a rapid-prototyping model for simulation surgery for radical resection and immediate reconstruction. Donor site was selected from parietal bone reflect shape, contour, and size of defect. Then radical resection of lesion and immediate reconstruction was performed as planned. Outcomes were assessed using clinical photographs and computed tomography scans. Successful reconstruction after radical resection was achieved by three-dimensional calvarial bone graft without complications. After a 12-month follow-up, sufficient bone thickness and symmetric soft tissue contour was well-maintained. By considering three-dimensional configuration of zygomaticomaxillary complex, the authors achieved satisfactory structural, aesthetic and functional outcomes without complications.
\end{abstract}

Keywords: Maxilla / Bone transplantation / Bone disease / Reconstructive surgical procedures

\section{INTRODUCTION}

Fibrous dysplasia (FD) is a bone disease which is characterized by abnormal proliferation and maturation of bone-forming stromal cells, resulting in replacement of normal bone structure with abnormal fibro-osseous connective tissue [1,2]. FD may involve of single bone (monostotic FD) or multiple bone (polyostotic FD). In monostotic FD, most commonly involved region is zygomaticomaxillary complex, which causes facial deformity/asymmetry, vision changes, hearing impairment, nasal

\section{Correspondence: Jong Won Hong}

Department of Plastic and Reconstructive Surgery, Yonsei University College of

Medicine, 50-1 Yonsei-ro, Seodaemun-gu, Seoul 03722, Korea

E-mail: hsaturn@hanmail.net

Received March 14, 2018 / Revised May 19, 2018 / Accepted May 24, 2018 congestion and/or obstruction, pain, paresthesia, and malocclusion [3]. Treatments for craniofacial FD include conservative shaving surgery or radical resection and immediate reconstruction [4]. In case of conservative shaving surgery, there is a potential risk of regrowth after contouring surgery and development of osteosarcoma in $1 \%$ of cases of monostotic FD [5]. In reconstruction methods, previous authors using autologous bone graft usually reconstruct zygoma as bar shape, less considering three-dimensional structure of zygoma $[3,6]$. To overcome these problems, we introduce the result of three-dimensional reconstruction with autologous calvarial bone graft after radical resection of FD in zygomaticomaxillary complex region, using rapid-prototyping $(\mathrm{RP})$ model. 


\section{CASE REPORT}

A 47-year-old male patient referred to Severance Hospital by incidentally detected FD of his left zygomaticomaxillary region after nasal trauma. On clinical examination, patient had facial asymmetry which had been developing for the past 10 years. His left malar eminence was more prominent then right side. Computed tomography (CT) image revealed monostotic proliferative fibrous lesion of zygomatic bone (Fig. 1). There were no symptoms of nerve compression, visual change or malocclusion. The patient wanted complete resection of FD due to possibility of recurrence or malignant change potential or sensory change.

$\mathrm{RP}$ model was produced from the $\mathrm{CT}$ scan with a 3D-printer (ProJet CJP 660Pro; 3D Systems, Rock Hill, SC, USA). Osteotomy line was drawn in RP model, while preserving infraorbital nerve (Fig. 2). Simulation surgery was performed in RP model. Donor site was determined from parietal bone considering not only size but also contour and shape of the zygomatic defect area by comparing three dimensional structures and curvature of zygoma to cranium. In RP model, calvarial bone was harvested and trimmed as tetrapod-like zygomatic defect shape. After harvesting calvarial bone, bihalving of outer cortex from inner cortex was done. Then, with outer cortex of calvarial bone graft, zygoma lesion was reconstructed in RP model.

After simulation surgery and planning in RP model, a patient underwent operation under general anesthesia. Bicoronal, sub- cilliary and gingivobuccal incisions were made to obtain adequate exposure and maximal visualization of field, eligible ac-

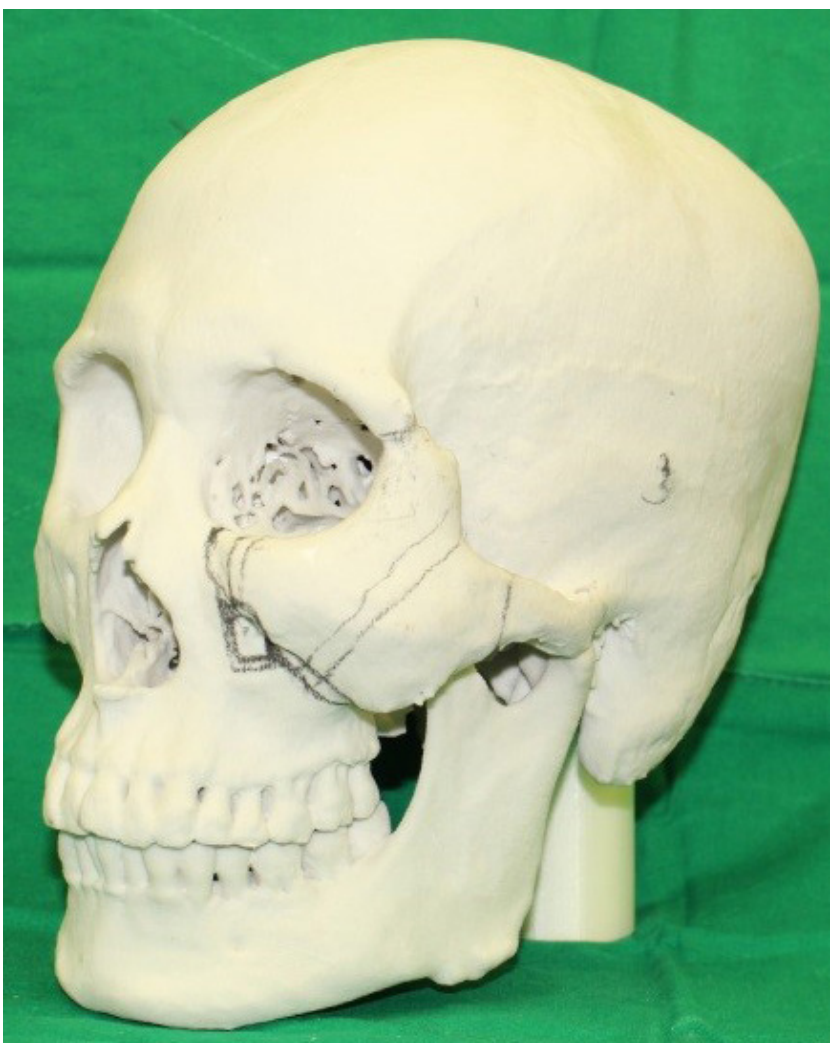

Fig. 2. Rapid prototyping model planning. Osteotomy line was drawn.
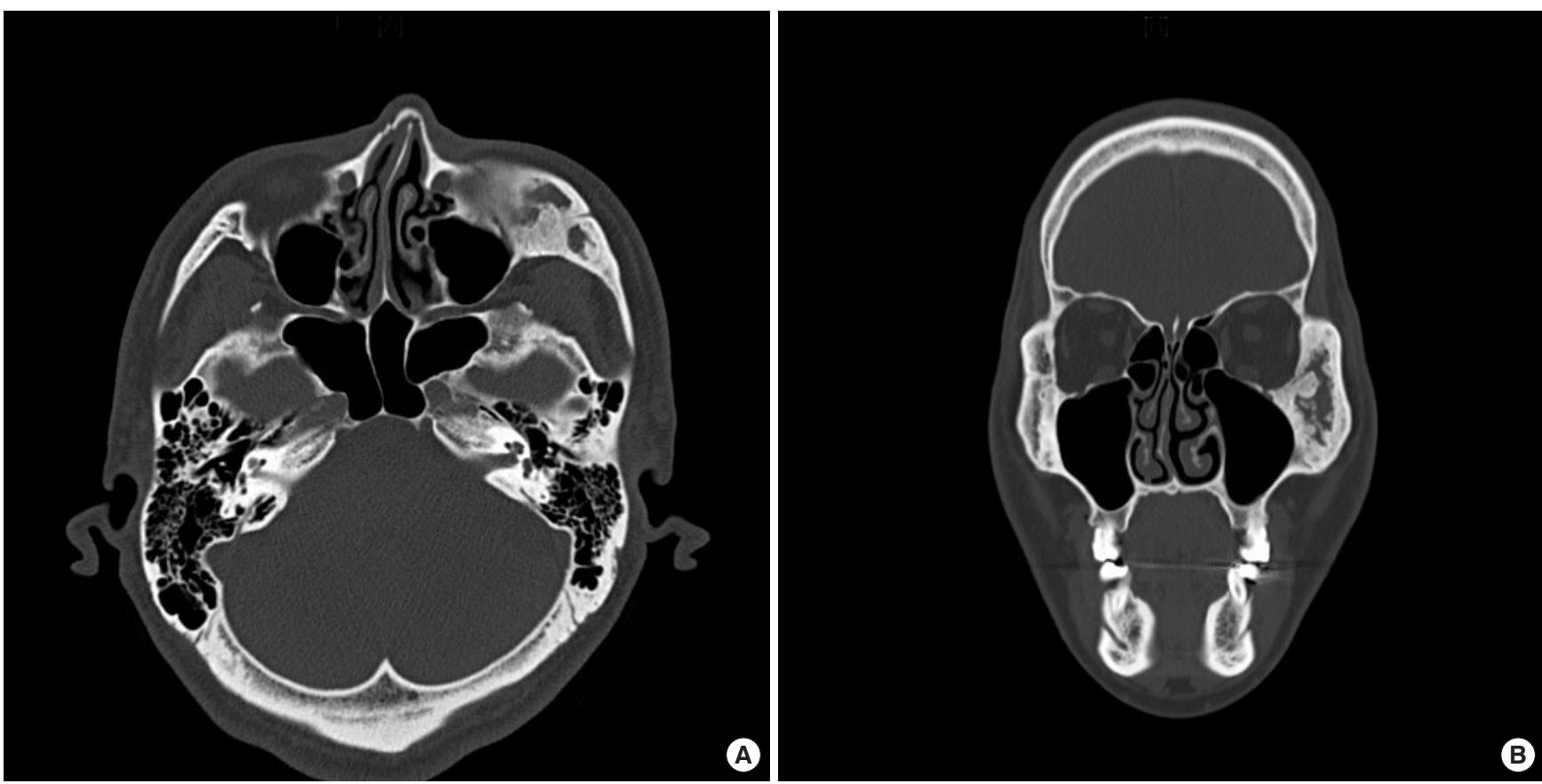

Fig. 1. Axial (A) and coronal (B) view of preoperative computed tomography image. Fibrous dysplasia is manifested in the left zygoma. 

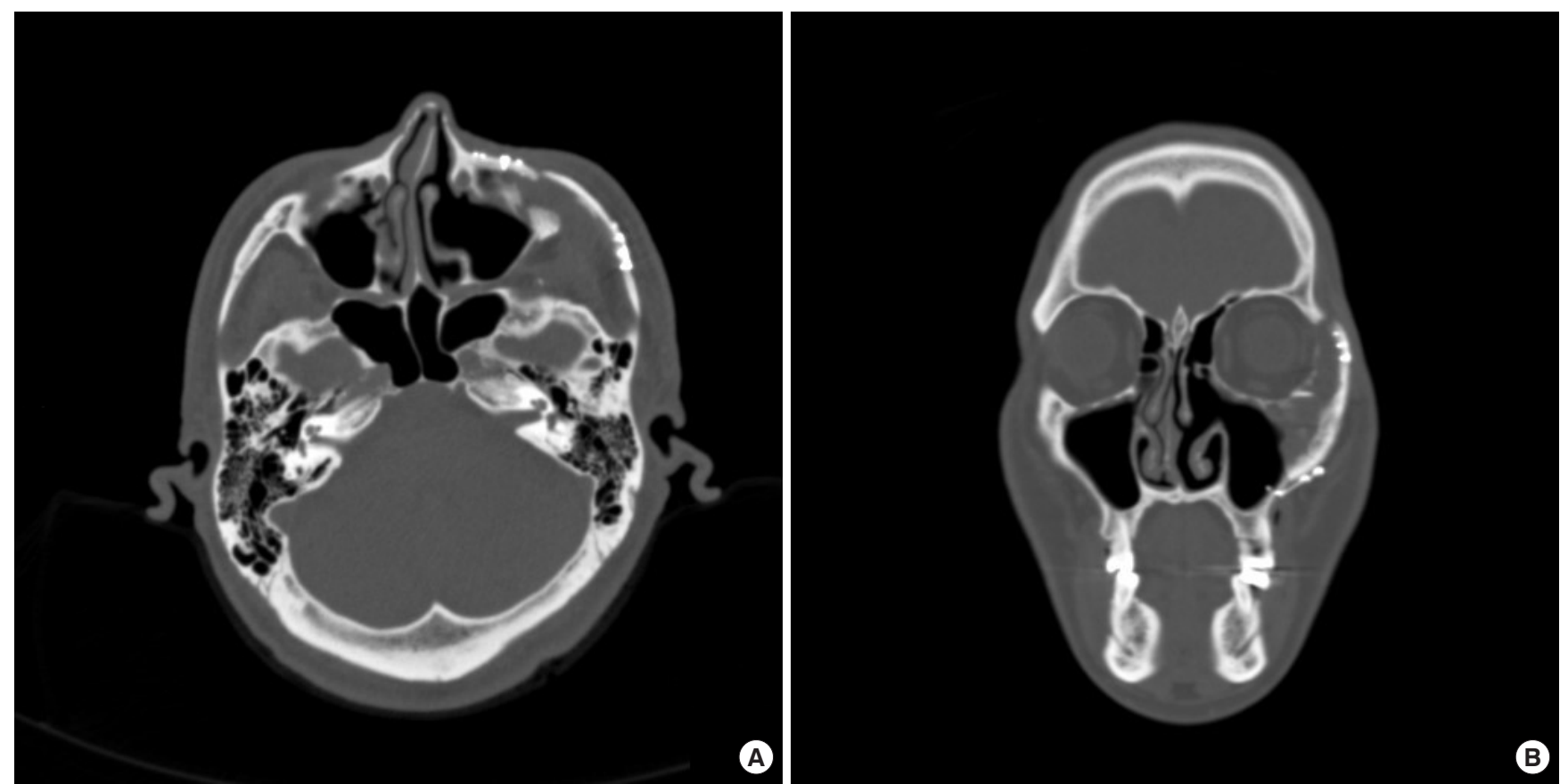

Fig. 3. Axial (A) and coronal (B) view of postoperative computed tomography image. Left zygoma was reconstructed with autologous calvarial bone graft.

cess to osteotomy and reconstruction. Planned osteotomy lines were marked. First, osteotomy began from infraorbital rim to lateral buttress of maxilla using reciprocating saw. Osteotomies in zygomatic arch and lateral orbital rim area were followed with oscillating saw. Finally, zygomatic bone was released from orbital floor and lateral orbital wall with osteotomies while carefully protecting eyeball and infraorbital nerve. Resected zygomatic bone was split to several pieces to pull out via gingivobuccal and bicoronal incision. Then, from left parietal bone, calvarial bone was harvested as oval shape. Then, bihalving of inner cortex and outer cortex was done. Inner cortex was fixed to donor site with dural tenting suture and titanium plate. Then, outer cortex was trimmed as bony defect shape. Then, trimmed calvarial bone graft was inserted via gingivobuccal incision to defect, and fixed from lateral orbital rim, zygomatic arch and lateral buttress with titanium plates and screws. After reconstructing the zygomatic bone, small bone segment was inserted to bony gap of orbital floor and lateral orbital wall and fixed with titanium plate. Then, lateral portion of periosteal layer underneath the orbicularis oculi muscle was anchored to the lateral orbital rim with suture to prevent lateral canthal deformity. Edges of the bone graft were burred to ensure a flush fit with no areas of prominence. Bicoronal, subcilliary and gingivobuccal incisions were closed with drain insertion. Endoscopic maxillary sinuplasty was performed to prevent sinusitis or mucocele by an otorhinolaryngologist. Patient was treated with radical resection and three-dimensional reconstruction with autologous calvarial bone graft. Clinical photography and CT scan was taken before and after surgery.

Postoperative outcomes were evaluated on clinical photographs and CT scans. On CT scans, reconstructed zygomatic bone maintained symmetric contour and sufficient bone thickness at a 12-month follow-up (Fig. 3). There was no evidence of recurrence of FD. When compared with preoperative clinical photographs, malar prominence was improved in postoperative 12-month photographs and CT image (Figs. 4, 5).

Hypoesthesia, secondary to zygomaticofacial nerve injury with zygomatic bone resection, was noticed on malar prominence area at postoperative 3 month, which totally recovered at postoperative 1 year. No other complications such as infection, facial nerve injury or visual symptoms were observed. Patient was satisfied with the result.

\section{DISCUSSION}

FD is mostly surgically intervened with two methods, conservative shaving or radical resection. Different craniofacial surgery groups reported their experience and suggested guidelines for optimal surgical intervention in FD. One of commonly accepted surgical indications is optic canal decompression with newly developed visual symptom [7]. Otherwise, decision for surgical approach should be based on age, affected craniofacial 


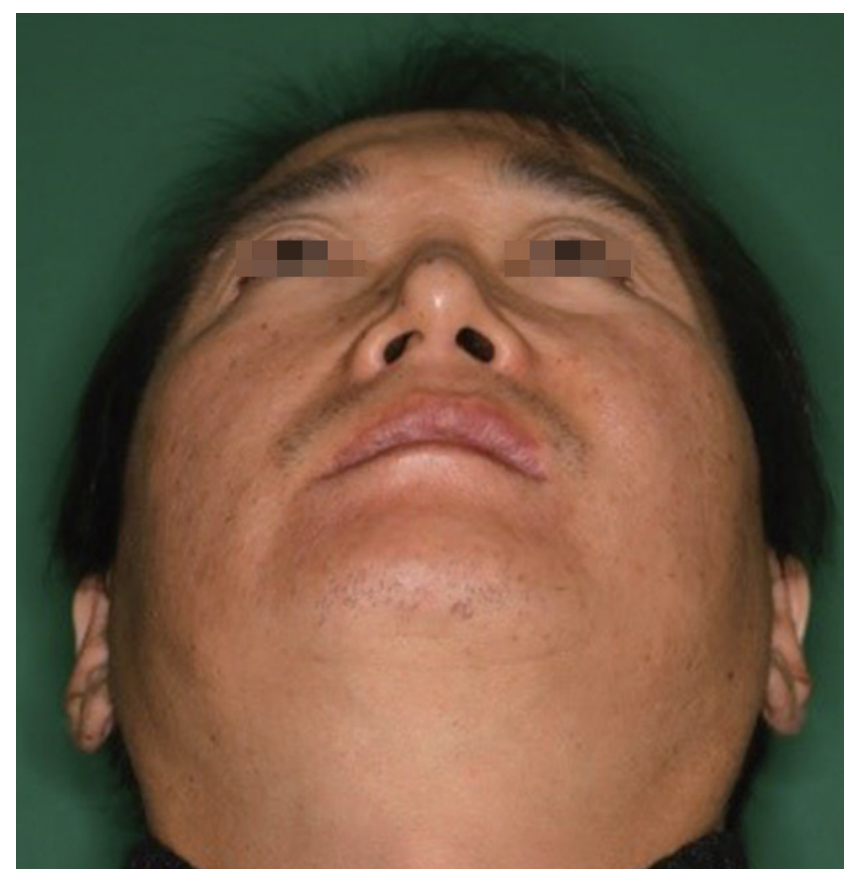

Fig. 4. Postoperative clinical photograph. Malar protrusion was improved after reconstruction.

bone site, extension of anatomical zone, functional and/or aesthetic issue, preferences of patient, and surgical team experience. Denadai et al. [8] suggested radical resection and immediate reconstruction for zone I, II, and IV in adult patient, while conservative shaving for younger age ( $<7$ years) without visual impairment. Because of potential risk of recurrence or malignant change, authors also prefer radical resection when possible in adult patients.

In a manner of reconstruction methods, previous authors usually described autologous bone graft with rib bone or calvarial split bone graft. Especially in zone 1, outer-table calvarial split bone grafts revealed as a reliable and safe methods with low complication rates $[4,9,10]$. However, in a previous operative technique of radical resection and immediate reconstruction with autologous calvarial bone or rib bone graft, original structure of zygomaticomaxillary complex as a three-dimensional configuration is less considered, and reconstructed only with bar-shape grafts for maxillary buttress stabilization $[3,6]$. To overcome this limitation, some authors suggest three-dimensional titanium mesh to reconstruct three dimensional configuration of zygoma $[11,12]$. Considering there are a potential risk of infection and exposure and increase preoperative procedural time and cost of customized titanium mesh, we prefer three-dimensional autologous calvarial bone graft.

In this report, by considering and reconstructing three-dimensional configuration of zygomaticomaxillary region, we achieved satisfactory structural and aesthetic outcomes without
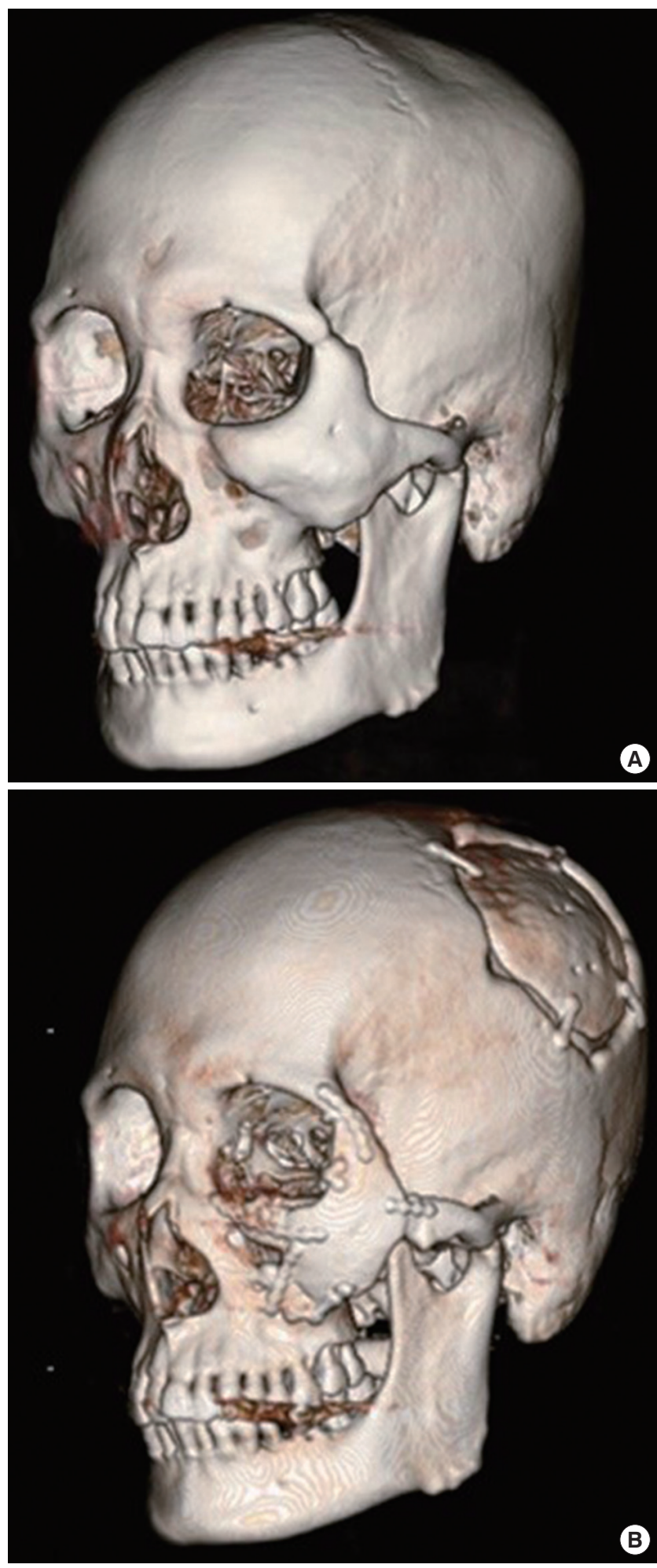

Fig. 5. Preoperative (A) and postoperative (B) computed tomography images. Left zygoma was reconstructed with autologous calvarial bone graft.

functional impairment or complications. We demonstrated three-dimensional reconstruction with autologous calvarial bone graft is eligible with proper planning with RP model. 


\section{CONFLICT OF INTEREST}

No potential conflict of interest relevant to this article was reported.

\section{PATIENT CONSENT}

The patients provided written informed consent for the publication and the use of their images.

\section{REFERENCES}

1. Riminucci M, Liu B, Corsi A, Shenker A, Spiegel AM, Robey PG, et al. The histopathology of fibrous dysplasia of bone in patients with activating mutations of the Gs alpha gene: sitespecific patterns and recurrent histological hallmarks. J Pathol 1999;187:249-58.

2. Hunt JA, Hobar PC. Common craniofacial anomalies: conditions of craniofacial atrophy/hypoplasia and neoplasia. Plast Reconstr Surg 2003;111:1497-508.

3. Valentini V, Cassoni A, Marianetti TM, Terenzi V, Fadda MT, Iannetti G. Craniomaxillofacial fibrous dysplasia: conservative treatment or radical surgery? A retrospective study on 68 patients. Plast Reconstr Surg 2009;123:653-60.

4. Chen YR, Chang CN, Tan YC. Craniofacial fibrous dysplasia: an update. Chang Gung Med J 2006;29:543-9.

5. Qu N, Yao W, Cui X, Zhang H. Malignant transformation in monostotic fibrous dysplasia: clinical features, imaging fea- tures, outcomes in 10 patients, and review. Medicine (Baltimore) 2015;94:e369.

6. Yang SJ, Choi JW, Chung YS, Ahn KM, Hong JP, Lee TJ, et al. Midfacial degloving approach for resectioning and reconstruction of extensive maxillary fibrous dysplasia. J Craniofac Surg 2012;23:1658-61.

7. Chen YR, Breidahl A, Chang CN. Optic nerve decompression in fibrous dysplasia: indications, efficacy, and safety. Plast Reconstr Surg 1997;99:22-30.

8. Denadai R, Raposo-Amaral CA, Marques FF, Ghizoni E, Buzzo CL, Raposo-Amaral CE. Strategies for the optimal individualized surgical management of craniofacial fibrous dysplasia. Ann Plast Surg 2016;77:195-200.

9. Powell NB, Riley RW. Facial contouring with outer-table calvarial bone: a 4-year experience. Arch Otolaryngol Head Neck Surg 1989;115:1454-8.

10. Smolka W, Eggensperger N, Kollar A, Iizuka T. Midfacial reconstruction using calvarial split bone grafts. Arch Otolaryngol Head Neck Surg 2005;131:131-6.

11. Lv M, Li J, Shen Y, Wang L, Sun J. The "drawer-like" resection and reconstruction with titanium mesh: a novel surgical technique for treatment of giant ossifying fibroma in the maxilla. J Oral Maxillofac Surg 2017;75:1752-61.

12. Shan XF, Chen HM, Liang J, Huang JW, Cai ZG. Surgical reconstruction of maxillary and mandibular defects using a printed titanium mesh. J Oral Maxillofac Surg 2015;73:1437. e1-9. 